\title{
An intensely pruritic disseminated skin eruption
}

A 64-year-old man presented to the dermatology outpatient clinic with a 4-month history of pruritic papules. He had a significant medical history of thyrotoxicosis, which was diagnosed 1 year prior and well controlled on carbimazole. He had no history of dyslipidaemia or underlying malignancy. The cutaneous eruption had first started on his abdomen, over his tattoo site. The tattoo was obtained 40 years prior, and he denied application of any topical medicaments over the area. The papules had been gradually expanding to include the rest of his chest, abdomen and back. Physical examination revealed multiple erythematous papules on his trunk that were moderately profuse. Some lesions were distributed in an annular configuration over his tattoo-naive chest and back, and the lesions did not correspond geographically to the pattern of his tattoo (Fig. 1).

Histological findings, diagnosis, treatment and progress. A biopsy of the papules revealed non-caseating granulomas, with focal necrobiosis and no giant cell inclusions (Fig. 2). Infective stains (periodic acid-Schiff stain, Ziehl-Neelsen stain, Grocott's methenamine silver stain and Wade-Fite stain) were negative. Dermal mucin was seen on Alcian blue staining. Other screening laboratory investigations for diabetes mellitus, hyperlipidaemia and human immunodeficiency virus were negative.

What is the most likely diagnosis?
A. Tattoo granuloma
B. Eruptive xanthomas
C. Molluscum contagiosum
D. Disseminated granuloma annulare
E. Cutaneous papular sarcoidosis

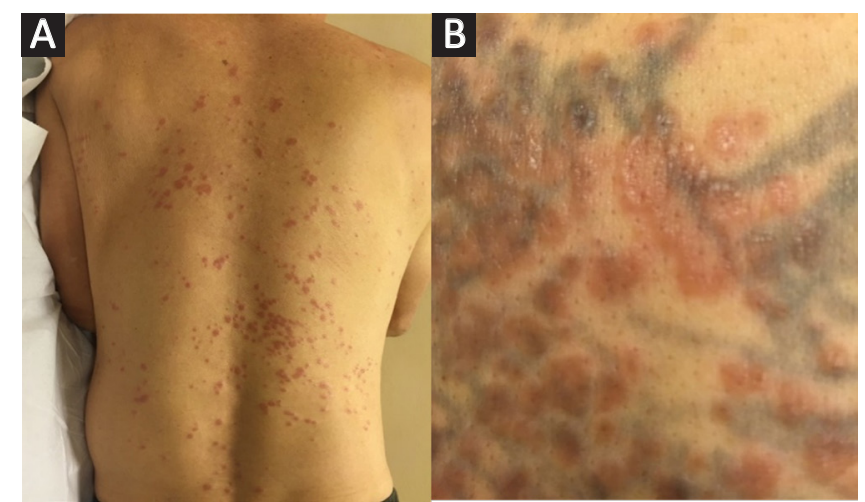

Fig. 1. Lesions distributed over the patient's (A) back and (B) abdomen.

A clinical diagnosis of disseminated granuloma annulare (DGA) was made. The patient was initially treated with potent topical steroids, such as clobetasol propionate cream and various antihistamines, without any improvement. After discussion with the patient, he was started on thrice-weekly narrow band ultraviolet B (NBUVB) phototherapy with a regime based on his skin phototype, starting from $400 \mathrm{~mJ} / \mathrm{cm}^{2}$. He demonstrated good temporal response to therapy with symptomatic reduction in pruritus, reduction in erythema and induration of the papules within the first month of phototherapy, and subsequent decrease in total body surface area affected. He tolerated the treatment well and was stepped down to twice-weekly phototherapy sessions after 3 months, and eventually stopped on resolution of his lesions at 6 months. His cumulative irradiation dose was $28.53 \mathrm{~J} / \mathrm{cm}^{2}$ over a total of 44 sessions. After cessation of NBUVB, he was followed up in the clinic with review sessions. He remained in remission with no further recurrence.

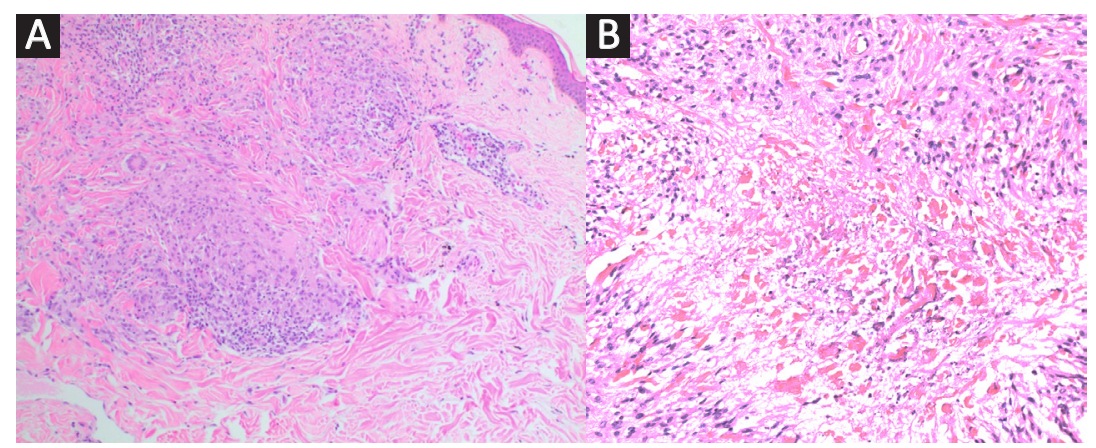

Fig. 2. (A) Non-caseating granulomas at 100x magnification on haematoxylin and eosin stain. (B) Focal necrobiosis and palisading granulomas at 400x magnification. 
Table 1. Summary of the patient's condition, clinical morphology and histology

\begin{tabular}{lll}
\hline Condition & Clinical morphology & Histology \\
\hline Tattoo granuloma & Papules and nodules occurring only at the site of a tattoo & Granulomas with pigment-laden macrophages \\
\hline $\begin{array}{l}\text { Eruptive xanthomas } \\
\text { Molluscum contagiosum }\end{array}$ & Yellow to skin-coloured papules, mostly on extensor surfaces & Foam cells and Touton giant cells in the dermis \\
\hline $\begin{array}{l}\text { Disseminated granuloma } \\
\text { annulare }\end{array}$ & $\begin{array}{l}\text { Widespread erythematous skin-coloured papules, usually } \\
\text { symmetrical and grouped }\end{array}$ & $\begin{array}{l}\text { Infundibular hyperplasia and papillomatosis with } \\
\text { central umbilication }\end{array}$ \\
\hline $\begin{array}{l}\text { Cutaneous papular } \\
\text { sarcoidosis }\end{array}$ & Erythematous skin-coloured dermal papules & lymphocytic infiltrates and increased dermal mucin \\
\hline
\end{tabular}

Discussion. DGA is uncommon, and unlike localised granuloma annulare that spontaneously resolves, it is particularly difficult to treat. While the underlying aetiology of DGA remains unknown, Mempel et al. postulated a T-cell-mediated immune response involving cytokines such as interleukin-2 (IL-2) to be the main drivers, with breakdown of matrix, elastic tissue and collagen by elastases, collagenases and metalloproteinases. ${ }^{1}$

DGA typically presents with groups of skin-coloured or erythematous dermal papules, and patients are commonly disturbed by its cosmetically obvious and widespread appearance, and unbearable pruritus. It is characterised by granulomas with lymphohistiocytic infiltrates on histology.

Treatment is guided by expert opinion and existing literature, which remains mostly limited to case reports. $^{2-6}$ Phototherapy has immunomodulatory effects, and has been proposed to play a role in the inhibition of T-lymphocytes, which are key in the pathogenesis of DGA. ${ }^{3}$ In addition, phototherapy is an advantageous modality over other systemic therapies such as isotretinoin, biologics or fumaric acid esters due to its relatively safer side effect profile. ${ }^{3}$ NBUVB has also been found superior to both broadband ultraviolet $B$ and ultraviolet $\mathrm{A}$ in the suppression of cytokine and lymphocyte proliferation - the suppression of which has been proposed to thwart granuloma formation in DGA. ${ }^{5,6}$ The role of NBUVB in the treatment of DGA in our context has also been similarly supported by previous case reports. ${ }^{6}$

Koebnerisation is a phenomenon that can be seen in both cutaneous sarcoidosis and tattoo granulomas, when lesions occur at previous sites of cutaneous injury. However, our patient's rash was not solely confined to the tattoo sites, and there were no temporal sequelae (Table 1).

Although eruptive xanthomas can also present as dermal papules, they are characteristically yellow in colour, and tend to present over the extensor surfaces, such as the elbows, knees and shoulders.
Tattoo granulomas are hypersensitivity reactions to the pigment used in tattoos. The condition typically manifests with granuloma formation as opposed to epidermal spongiosis, which is typically seen in contact dermatitis on histology.

Molluscum contagiosum, on the other hand, is a cutaneous infection by the poxvirus. It presents with distinctive waxy papules with central umbilicated pits, and intracytoplasmic inclusion bodies with infundibular hyperplasia and papillomatosis on histology.

\section{REFERENCES}

1. Mempel M, Musette P, Flageul B, et al. T-cell receptor repertoire and cytokine pattern in granuloma annulare: defining a particular type of cutaneous granulomatous inflammation. J Invest Dermatol 2002;118:957-66.

2. Lukács J, Schliemann S, Elsner P, et al. Treatment of generalized granuloma annulare - a systematic review. J Eur Acad Dermatol Venereol 2015;29:1467-80.

3. Muylaert BPB, Almada R, Vasconcelos RCF. Granuloma annulare treated with narrowband UVB phototherapy. An Bras Dermatol 2017;92(5 Suppl 1):82-4.

4. Tjioe M, Smits T, van de Kerkhof PC, et al. The differential effect of broad band vs narrow band UVB with respect to photodamage and cutaneous inflammation. Exp Dermatol 2003;12:729-33.

5. Cunningham L, Kirby B, Lally A, et al. The efficacy of PUVA and narrowband UVB phototherapy in the management of generalised granuloma annulare. J Dermatolog Treat 2016;27:136-9.

6. Yong A, Chong WS, Pan JY. Disseminated granuloma annulare responding to narrowband UVB phototherapy. Photodermatol Photoimmunol Photomed. 2016;32:107-9.

Laura LY $\underline{\text { Hui }}{ }^{1}{ }_{M C R P}$, Michelle MF $\underline{\text { Chan }}{ }^{2}$ FRCPath, Shiu Ming Pang ${ }^{1,3}$ FRCP (London)

\footnotetext{
${ }^{1}$ Department of Dermatology, Singapore General Hospital, Singapore

2 Department of Anatomical Pathology, Singapore General Hospital, Singapore

${ }^{3}$ Duke-NUS Medical School, Singapore
}

Correspondence: Dr Laura LY Hui, Department of Dermatology, Singapore General Hospital, Outram Road, Block 6 Level 9, Singapore 169608.

Email: lauraahui@gmail.com 